\title{
Implementación de material educativo gamificado para la enseñanza-aprendizaje de la matemática en alumnos con Síndrome de Down
}

\author{
Implementation of gamified educational material for the teaching-learning \\ of mathematics in students with Down Syndrome
}

Recibido: 30/09/2019

Aceptado: $18 / 02 / 2020$

Publicado: 01/06/2020

\author{
Nancy Castillo Jumbo \\ Universitat Rovira i Virgili \\ nancydolores.castillo@estudiants.urv.cat \\ José Jiménez González \\ Universitat Rovira i Virgili \\ josemiguel.jimenez@urv.cat
}

\section{RESUMEN}

Esta investigación examina la incidencia que tiene la implementación de una propuesta didáctica de aula basada en Recursos Educativos Gamificados (REG), en el rendimiento académico (RA) de los niños/jóvenes con Síndrome de Down, en la asignatura de matemática; estableciendo las estrategias metodológicas que facilitan el aprendizaje de los primeros conceptos matemáticos (conteo, las nociones de cantidad, suma y resta simples) y demostrar que el uso de recursos educativos gamificados (juegos) y metodologías estructuradas pueden mejorar su competencia en el aprendizaje de la matemática. Para ello, se utilizan siete juegos que se trabajaron con tres alumnos con Síndrome de Down de entre 9 y 22 años, se presentan los estudios de caso de cada uno de ellos. Durante la implementación de los recursos educativos gamificados, se realiza la evaluación inicial (pretest) y final (postest) de los alumnos mediante el Test de Habilidades Básicas para la Iniciación al Cálculo (Riquelme,2003), el mismo que demuestra avances en las habilidades. Las técnicas estadísticas utilizadas para analizar los datos son t-students y análisis de varianza (ANOVA) para comparar puntuaciones medias. Específicamente, los alumnos con Síndrome de Down tras el análisis de resultados demuestran mejora significativa del rendimiento académico en el aprendizaje de conocimientos y habilidades relacionadas con la clasificación, seriación, conservación, expresión de juicio lógico y función simbólica, aunque los mejores resultados están en las habilidades de clasificación, seriación y juicio lógico, no así, en la expresión de conservación y función simbólica donde se observa una mejora moderada.

\section{PALABRAS CLAVE}

Rendimiento; Síndrome de Down; material educativo; enseñanza.

\section{ABSTRACT}

This research examines the impact of the implementation of a classroom didactic proposal based on Gamified Educational Resources (REG), on the academic performance (AR) of children and/or young people with Down Syndrome, in the subject of mathematics; establishing the methodological strategies that facilitate the learning of the first mathematical concepts (counting, the notions of quantity, simple addition and subtraction) and demonstrate that the use of gamified educational resources (games) and structured methodologies can improve their competence in learning the mathematics. For this, seven games were used, which were worked with three students with Down Syndrome between 9 and 22 years, the case studies of each of them are presented. During the implementation of the gamified educational resources, the initial (pretest) and final (post-test) evaluation of the students is carried out through the Basic Skills Test for the Introduction to Calculus (Riquelme, 2003), which demonstrates advances in skills. The statistical techniques 
used to analyze the data are t students and analysis of variance (ANOVA) to compare average scores. Specifically, students with Down Syndrome after the analysis of results demonstrate significant improvement in academic performance in learning knowledge and skills related to classification, seriation, conservation, expression of logical judgment and symbolic function, although the best results are in the classification, seriation and logical judgment skills, not so, in the expression of conservation and symbolic function where a moderate improvement is observed.

\section{KEYWORDS}

Performance; Down's Syndrome; educational material; teaching.

\section{CITA RECOMENDADA}

Castillo, N. y Jiménez, J. (2020). Implementación de material educativo gamificado para la enseñanza-aprendizaje de la matemática en alumnos con Síndrome de Down. Revista Interuniversitaria de Investigación en Tecnología Educativa, 8, 1-13. https://doi.org/10.6018/riite.397741

\section{Principales aportaciones del artículo y futuras líneas de investigación:}

- Presenta herramientas, técnicas y estrategias gratuitas que permiten ayudar a los estudiantes con Síndrome de Down en el avance de su aprendizaje de la matemática.

- Evidenciar que el apoyo familiar y su nivel socioeconómico, son una ventaja para que los estudiantes ya usen herramientas tecnológicas y por otro lado la atención temprana

- Futura línea de investigación: Diversidad, inclusión y tecnología.

\section{INTRODUCCIÓN}

Para crear y/o utilizar herramientas y material educativo destinado a personas con Síndrome de Down es importante investigar las características principales de su proceso de aprendizaje. Según algunos estudios Bautista (2010) y otros autores, citados por Tangarife \& Jaramillo (2015), señala que, estas características son: aprendizaje más lento, comparado al de las personas con desarrollo normal; requieren mayor número de ejemplos del concepto enseñado; aprenden mejor cuando se utilizan métodos visuales; algunos requieren el apoyo de profesionales en pedagogía terapéutica y audición; presentan problemas para mantener la atención, entre otras.

Por otro lado, Down España (2012), señala que los resultados del Proyecto H@z Tic sostienen que hoy día:

Las Tecnologías de la Información y de Comunicación (TIC) han demostrado tener un gran potencial para el aprendizaje y la inclusión social de las personas con Síndrome Down. Estas tecnologías ayudan a desarrollar la memoria semántica, relacionada con el significado de las palabras y el conocimiento, centran la atención, aumentando la capacidad de almacenamiento y de procesamiento de la información, posibilitan una mejor comprensión de lo abstracto, refuerzan la visión y la audición; y, corrigen trastornos de lenguaje. (pág. 13)

Entonces, para incorporar el uso de las TIC como herramientas pedagógicas en los procesos educativos se debe realizar algunas adaptaciones curriculares específicamente para los estudiantes con Necesidades Educativas Especiales (NEE). Esto revela que es necesario investigar sobre las características y necesidades educativas de las personas con algún tipo de discapacidad, para contribuir a mejorar la calidad de la respuesta educativa, especialmente en la adopción de estrategias metodológicas acordes a las características cognitivas y estilo de aprendizaje de esta población y en el momento de realizar adaptaciones del currículo. 
Finalmente, es importante mencionar que la elección del tema del uso de recursos educativos gamificados (juegos) y el desarrollo de habilidades para el cálculo en los alumnos con síndrome de Down, ha sido por la necesidad presentada en nuestro país de atender a este grupo prioritario a fin de conocer sobre ¿Cómo se pueden trabajar contenidos curriculares con estos recursos? ¿Cuáles son criterios para seleccionar los recursos más adecuados? y ¿De qué manera debe actuar el docente?

\subsection{Objetivos de investigación}

\subsubsection{General}

Validar materiales educativos gamificados para la enseñanza-aprendizaje de la matemática en niños/jóvenes con Síndrome de Down de la Escuela de Educación Básica Especializada "Padre Franco Aguirre"

\subsubsection{Específicos}

- Implementar recursos educativos(juegos) para la adquisición del conocimiento matemático a fin de establecer las estrategias metodológicas que facilitan el aprendizaje de los primeros conceptos matemáticos (conteo, las nociones de cantidad, suma y resta simples)

- Demostrar que el uso de materiales educativos gamificados y metodologías estructurados puede mejorar la competencia en el aprendizaje de la matemática de los niños/jóvenes con Síndrome de Down

- Contribuir a la mejora de la oferta de educación especializada en el área de matemática que brindan los docentes, psicoterapeutas y psicólogos a los niños/jóvenes con Síndrome Down en la Escuela de Educación Básica Especializada "Padre Franco Aguirre".

\subsection{Estado del arte}

Hablar de Educación para todos y todas es hablar de Inclusión; la Atención a la Diversidad requiere respeto, es fuente de riqueza y oportunidad para el aprendizaje. Las personas tenemos cosas que aportar en la escuela, en la familia o en la comunidad: somos igualmente válidos. Por lo tanto, "un Sistema Educativo Inclusivo debe garantizar la igualdad de oportunidades para todo el alumnado, independientemente de sus condiciones personales, económicas, sociales o culturales" (Antequera et al., 2008). Esta lucha por la igualdad de oportunidades se hace aún más necesaria y conveniente para con el alumnado con necesidades específicas de apoyo educativo y para con el alumnado con diversidad funcional Intelectual, objeto de atención de la presente investigación. Específicamente nos centraremos en la atención al alumnado con Síndrome Down:

Un grupo de escolares que demanda una atención educativa especializada, con planes de actuación personalizados, entendidos como un continuo sustentado en tres pilares principales: la detección de las necesidades educativas especiales lo más tempranamente posible; la valoración del grado de desarrollo real y potencial de sus capacidades; y, por último, la toma adecuada de decisiones para organizar la atención educativa según las necesidades, estableciéndose así la modalidad de escolarización oportuna y la determinación de los apoyos y recursos ordinarios y/o especializados más idóneos según el caso (Angulo, et al., 2008, p. 5).

Esta investigación se centra en la atención al alumnado con síndrome Down a través del uso de materiales educativos gamificados más adecuados y teniendo en cuenta su forma de ser y aprender. Toda esta propuesta encierra, en definitiva, la finalidad de proporcionar un marco general que sirva como referente a la comunidad educativa a la hora de proyectar una respuesta adecuada, realizar una valoración y posterior seguimiento de la misma. Y todo ello, con el propósito que cada uno de estos escolares consiga el mayor nivel de desarrollo posible de sus potencialidades.

Por tanto, para alcanzar una calidad educativa que dé respuesta a las necesidades educativas de todos los estudiantes es necesario un cambio en la gestión escolar para conducir 
a la transformación de prácticas pedagógicas en atención a las necesidades educativas especiales. Se considera ésta como una situación compleja donde los docentes no cuentan con estrategias adecuadas para dar respuesta de manera efectiva, lo que se constituye en una necesidad urgente a resolver. Se pretende entregar orientaciones y guiar las acciones que deben realizar los docentes en la atención a estudiantes con Síndrome de Down y contribuir al cumplimiento del reto del Ministerio de Educación del Ecuador de proporcionar una cultura común a todos los estudiantes, que evite la discriminación y desigualdad de oportunidades, respetando al mismo tiempo sus características y necesidades individuales.

\subsubsection{La educación de los niños con Síndrome Down}

La educación es una pieza fundamental de la que disponen las personas con discapacidad para su crecimiento integral, ya que en la mayoría de los casos es el elemento que mayor impacto tiene para su crecimiento y realización personal (Federación Andaluza de Asociaciones para el Síndrome de Down, 2012). Por ello, debemos seleccionar al máximo todos los recursos y agentes involucrados en el proceso educativo: los métodos de enseñanza y aprendizaje, TIC, el profesorado, las instituciones educativas, las familias, el entorno social; así como los formatos en los que el estudiante recibe los conocimientos, ya que de todo ello dependerá su desarrollo intelectual, social y humano.

Considerando algunas características psicológicas y de aprendizaje propias de las personas con discapacidad intelectual los resultados del Proyecto H@z Tic (Down España, 2012) sostienen que las TIC ayudan a retener, evocar y relacionar distintas informaciones, mejoran la generalización y el mantenimiento del aprendizaje, fomentan la iniciativa para comenzar actividades y la constancia para realizar tareas menos motivadoras, favorecen la reflexión amentando la memoria visual, lo cual facilita un aprendizaje más rápido con el apoyo de imágenes. Por tanto, es importante realizar algunas adaptaciones curriculares, de tal forma que incorporen el uso de las TIC como herramientas pedagógicas en los procesos educativos, principalmente en las poblaciones con NEE. Además, según afirman Serna et al. (2005), los estudiantes con Síndrome de Down tienen un potencial de aprendizaje y de desarrollo que, con los apoyos y ayudas necesarios, les permite alcanzar elevados niveles de logro en los aprendizajes escolares, en su capacidad de desenvolvimiento autónomo y una adecuada inserción social y laboral.

El uso de las TIC en la labor docente es uno de los aspectos en los que se enfatiza. Su incuestionable incorporación al espacio educativo no debe servir para conservar los viejos modos de enseñar, sino para la adecuación de los mismos a los nuevos espacios digitales en los que se está desarrollando el mundo. El objetivo perseguido con el uso de los juegos y videojuegos en la enseñanza es que los alumnos aprendan más, aprendan mejor y disfruten aprendiendo, así como el desarrollo de una aplicabilidad real y la resolución de problemas de la vida cotidiana.

En este contexto surge y aumenta el uso de la tecnología en la escuela, y una de sus vertientes más innovadoras actualmente es la gamificación. Esta estrategia ha mostrado su eficacia, hasta ahora, en ejercicios empresariales y recientemente están surgiendo diversas experiencias aplicadas a la educación (Zicherman \& Cunningham, 2011). Precisando a la gamificación como:

El uso de estrategias, modelos, dinámicas, mecánicas y elementos propios de los juegos en contextos ajenos a éstos, con el propósito de transmitir un mensaje o unos contenidos o de cambiar un comportamiento, a través de una experiencia lúdica que propicie la motivación, la implicación y la diversión. (Llorens-Largo et al., 2016, p.25)

A continuación, se presenta una tabla resumen con la información más relevante sobre los resultados de experiencias basadas en gamificación, organizados por fecha de publicación y autoría, los objetivos, la muestra de cada estudio, la intervención realizada y sus resultados.

Tabla 1. Experiencias basadas en Gamificación para la enseñanza de la matemática en Alumnado con Síndrome de Down.

\begin{tabular}{lllll}
\hline Autor y Año & Objetivos & Muestra & Intervención & Resultados \\
\hline
\end{tabular}




\begin{tabular}{|c|c|c|c|c|}
\hline $\begin{array}{l}\text { Bonilla, } \\
\text { (2012) }\end{array}$ & $\begin{array}{l}\text { Utilizar el software } \\
\text { LOGO, para } \\
\text { ayudar a los niños } \\
\text { con Síndrome } \\
\text { Down para } \\
\text { aprender } \\
\text { contenidos lógico- } \\
\text { matemático, en } \\
\text { particular en } \\
\text { relación al conteo } \\
\text { y la noción de } \\
\text { cantidad. }\end{array}$ & $\begin{array}{l}\text { Grupo de control } \\
\text { ( } 2 \text { alumnos) que } \\
\text { asistían a } \\
\text { escuela regular } \\
\text { en primer grado } \\
\text { de primaria }\end{array}$ & $\begin{array}{l}\text { Diseñar e } \\
\text { implementar una } \\
\text { secuencia de } \\
\text { actividades } \\
\text { computacionales } \\
\text { creados en } \\
\text { MSWLOGO, que } \\
\text { faciliten el } \\
\text { aprendizaje del } \\
\text { conteo y del } \\
\text { concepto de cantidad } \\
\text { en niños con } \\
\text { Síndrome Down }\end{array}$ & $\begin{array}{l}\text { Los resultados, } \\
\text { confirman que en } \\
\text { términos generales, } \\
\text { los alumnos con } \\
\text { Síndrome Down } \\
\text { mostraron ciertos } \\
\text { avance en el } \\
\text { desarrollo de sus } \\
\text { conocimientos y } \\
\text { habilidades } \\
\text { relacionadas al } \\
\text { conteo, aunque en el } \\
\text { grupo de control el } \\
\text { avance fue mucho } \\
\text { más notorio, aunque } \\
\text { partió de un nivel de } \\
\text { conocimientos previos } \\
\text { más altos. }\end{array}$ \\
\hline $\begin{array}{l}\text { Pareja, } \\
(2015)\end{array}$ & $\begin{array}{l}\text { Dar respuesta al } \\
\text { interés del } \\
\text { profesorado } \\
\text { sobre el } \\
\text { aprovechamiento } \\
\text { de tablets, y } \\
\text { dispositivos } \\
\text { móviles } \\
\text { (MLearning) } \\
\text { como } \\
\text { recursos } \\
\text { educativos, } \\
\text { además de } \\
\text { nuevas } \\
\text { tendencias } \\
\text { didáctico } \\
\text { formativas } \\
\text { como la aplicación } \\
\text { de los videojuegos } \\
\text { en el aula. }\end{array}$ & $\begin{array}{l}\text { Dar respuesta al } \\
\text { interés del } \\
\text { profesorado } \\
\text { sobre el } \\
\text { aprovechamiento } \\
\text { de tablets, y } \\
\text { dispositivos } \\
\text { móviles } \\
\text { (MLearning) } \\
\text { como } \\
\text { recursos } \\
\text { educativos, } \\
\text { además de } \\
\text { nuevas } \\
\text { tendencias } \\
\text { didáctico } \\
\text { formativas } \\
\text { como } \\
\text { aplicación de los } \\
\text { videojuegos } \\
\text { en el aula. }\end{array}$ & $\begin{array}{l}\text { Implementación de } \\
\text { una App creada por } \\
\text { la autora que, } \\
\text { mediante el uso de } \\
\text { las mecánicas del } \\
\text { juego involucra al } \\
\text { alumnado con } \\
\text { síndrome } \\
\text { de Down en la } \\
\text { resolución de } \\
\text { problemas, para } \\
\text { desarrollar el } \\
\text { pensamiento lógico y } \\
\text { reflexivo. }\end{array}$ & $\begin{array}{l}\text { La implementación de } \\
\text { la App, demuestra ser } \\
\text { un recurso que obtiene } \\
\text { valores altos en los } \\
\text { cuestionarios pasados } \\
\text { a profesorado y } \\
\text { alumnado } \\
\text { (puntuaciones entre } 4 \\
\text { y } 5 \text { sobre } 5 \text { ). Se } \\
\text { destaca que el } \\
\text { contenido es ameno y } \\
\text { que el esfuerzo del } \\
\text { alumnado se ve } \\
\text { reflejado en buenos } \\
\text { resultados. }\end{array}$ \\
\hline $\begin{array}{l}\text { Tangarife \& } \\
\text { Jaramillo } \\
\text { (2015) }\end{array}$ & $\begin{array}{l}\text { Presenta los } \\
\text { resultados } \\
\text { obtenidos con } \\
\text { "una App, } \\
\text { diseñada y } \\
\text { desarrollada con } \\
\text { el objetivo } \\
\text { principal de } \\
\text { facilitar la } \\
\text { enseñanza de las } \\
\text { operaciones } \\
\text { básicas de las } \\
\text { matemáticas a } \\
\text { personas con } \\
\text { SD". }\end{array}$ & $\begin{array}{l}\text { Cuatro } \\
\text { aprendices y dos } \\
\text { de ellos solo } \\
\text { participaron en } \\
\text { una sesión } \\
\text { (Fundación Casa } \\
\text { Taller Artesas } \\
\text { (Medellín, } \\
\text { Colombia)) }\end{array}$ & $\begin{array}{l}\text { "La aplicación está } \\
\text { diseñada para } \\
\text { funcionar } \\
\text { principalmente en } \\
\text { dispositivos como } \\
\text { Smartphones y } \\
\text { tablets, también } \\
\text { puede ser instalada } \\
\text { en computadores de } \\
\text { escritorio. Se } \\
\text { realizaron dos } \\
\text { sesiones en dos días } \\
\text { en las que se } \\
\text { desarrollaron } \\
\text { actividades con los } \\
\text { módulos de: } \\
\text { ordenamiento, } \\
\text { agrupamiento, suma, } \\
\text { ejercicios de suma, } \\
\text { resta y ejercicios de } \\
\text { resta. Las sesiones } \\
\text { tuvieron una } \\
\text { duración de } 60 \text { a } 80 \\
\text { minutos". }\end{array}$ & $\begin{array}{l}\text { "La aplicación tiene } \\
\text { una gran aceptación y } \\
\text { motivación de los } \\
\text { aprendices. Con el } \\
\text { uso de la aplicación y } \\
\text { por realizar cada una } \\
\text { de las actividades, } \\
\text { solo en uno de ellos } \\
\text { se observó alguna } \\
\text { dificultad en el uso de } \\
\text { la pantalla táctil en las } \\
\text { actividades de } \\
\text { ordenamiento y } \\
\text { agrupamiento, al } \\
\text { arrastrar los objetos y } \\
\text { levantar el dedo sin } \\
\text { haberlo llevado hasta } \\
\text { la posición final”. }\end{array}$ \\
\hline
\end{tabular}




\begin{tabular}{|c|c|c|c|c|}
\hline $\begin{array}{l}\text { (Alonso, } \\
2016)\end{array}$ & $\begin{array}{l}\text { Realiza una } \\
\text { aplicación en la } \\
\text { nube } \\
\text { Juegamáticas que } \\
\text { ayuda a las } \\
\text { personas con } \\
\text { Síndrome Down a } \\
\text { reforzar su } \\
\text { aprendizaje y } \\
\text { conocimientos } \\
\text { matemáticos } \\
\text { empleando un } \\
\text { sistema de } \\
\text { gamificación } \\
\text { guiado. }\end{array}$ & $\begin{array}{l}\text { Una chica (18 } \\
\text { años) y un chico } \\
\text { (13 años) }\end{array}$ & $\begin{array}{l}\text { Este proyecto } \\
\text { multidisciplinar aplica } \\
\text { diferentes } \\
\text { metodologías tanto } \\
\text { para el desarrollo de } \\
\text { actividades (tipos de } \\
\text { ejercicios, niveles, } \\
\text { orden de los } \\
\text { mismos). Este se ha } \\
\text { desarrollado en la } \\
\text { plataforma Google } \\
\text { App Engine, basada } \\
\text { en prototipos }\end{array}$ & $\begin{array}{l}\text { Resultados } \\
\text { satisfactorios en el } \\
\text { uso de las } \\
\text { aplicaciones con } \\
\text { problemas como } \\
\text { puzles, posiciones en } \\
\text { el espacio y de orden } \\
\text { de tamaño, solamente } \\
\text { indican que un } \\
\text { aspecto negativo es } \\
\text { que Google ha dejado } \\
\text { un poco descuidados } \\
\text { los proyectos GWT y } \\
\text { Objectify en algunos } \\
\text { aspectos. }\end{array}$ \\
\hline
\end{tabular}

\section{MÉTODO}

\subsection{Participantes}

El contexto educativo y población en el que se enmarca esta investigación está compuesto por todos estudiantes con Síndrome Down de la Escuela de Educación Básica Especializada "Padre Franco Aguirre" a la cual asisten 36 estudiantes regularmente en año lectivo 2018-2019, cuenta con 4 docentes y un líder educativo. De acuerdo a información suministrada por la Unidad Distrital de Apoyo a la Inclusión del Distrito 11D06. Los datos de los participantes son: el primero de ellos cursa el 9no grado con 22 años de edad, mientras que la otra estudiante cursa el $7 \mathrm{mo}$ grado con 14 años de edad y la última estudiante cursa el 5to año de educación básica con 9 años de edad. Respecto al género 2 son mujeres, es decir, el $66.6 \%$ y $33.3 \%$ son hombres.

\subsection{Enfoque y diseño metodológico}

Una vez revisada bibliografía acerca del tema, se considera que la investigación se adapta a la Investigación Basada en Diseño en el campo de la Tecnología Educativa, ya que se trata de responder a la necesidad de inclusión educativa de los niños/jóvenes con Síndrome Down. Una realidad educativa que para Plomp (2010) citado por De Benito \& Salinas (2016), requiere:

Un estudio sistemático de diseñar, desarrollar y evaluar intervenciones educativas (ya sean programas, estrategias, o los materiales de enseñanza-aprendizaje, productos y sistemas) como soluciones a problemas complejos de la práctica educativa, que al mismo tiempo tiene por objeto la mejora de nuestro conocimiento sobre las características de estas intervenciones y sobre los procesos de diseño y desarrollo de las mismas. (p.3)

En esta investigación es la introducción de materiales educativos gamificados para que sean sometidos a pruebas y validación, y, una vez mejorados, se puedan difundir a la realidad escolar.

\subsection{Diseño del Procedimiento de investigación y técnicas de recogida de datos}

Para el desarrollo de la investigación, se llevaron a cabo cuatro fases:

Fase de definición del problema/análisis/exploración: análisis de la situación actual de los niños y/o jóvenes en el Sistema Educativo Ecuatoriano con la finalidad de definir las necesidades/problema, luego se realiza la búsqueda documental para el desarrollo de la fundamentación teórica del estudio en base a artículos, libros e investigaciones sobre el tema para el desarrollo/selección de soluciones de acuerdo a la fundamentación teórica.

Fase de diseño/prototipado/desarrollo: desarrollo de prototipo de intervención, en base a fundamentación teórica se desarrolla: encuesta a profesores para conocer formas de aprendizaje de estudiantes; rubrica para seleccionar los recursos educativos. El tipo de investigación es 
cuasi experimental de pruebas que permiten diferenciar las medias obtenidas de las pruebas de habilidades para el cálculo en el grupo de estudiantes antes y después de la intervención.

Implementación con la realización de la evaluación inicial a los niños con el fin de encontrar las diferencias significativas entre los diferentes grupos. Se utilizará la Escala de Inteligencia de L.M. Terman y M.A. Merrill. que, según señala Ruiz (2012), es la prueba que mejor discrimina, ya que permite obtener un C.I. hasta con niveles muy bajos de capacidad y proporciona una puntuación de edad mental. Para analizar el conteo y precantidad se les aplicará el Test de Habilidades Básicas para la Iniciación al Calculo "TIC" que es una herramienta útil para que los educadores realicen un buen diagnóstico (Pretest), como también adecuar los procesos de enseñanza y de aprendizaje a las realidades observadas. La estructura de la prueba está constituida por 32 ítems, los que están organizados en cinco habilidades-dimensiones: clasificación, seriación, conservación, expresión de juicio lógico y función simbólica (Riquelme, 2003). Para la recogida de datos, se utilizan siete juegos adaptados a sus características. Son juegos que tienen aspectos de estrategia y de conocimiento, ya que generen situaciones problemáticas para cuyo abordaje son necesarios conceptos y técnicas presentes en el currículo y, al mismo tiempo, su práctica promueve el descubrimiento y aplicación de estrategias. Construcción del plan de Intervención, mediante la interacción de los alumnos con los juegos queremos observar si utilizan y/o tienen adquirido conocimientos trabajados anteriormente con otros recursos y qué estrategias utilizan. Llevar a cabo 3 sesiones con cada una de los juegos, para un total de 21 sesiones con cada alumno. Durante las sesiones de trabajo se interactuará, de manera individual, con cada uno de los tres alumnos con SD (Diego, Mayra y Marilyn) en la Institución Educativa del estudio.

Evaluación final de los niños para observar si existieron cambios significativos con respecto a los conocimientos de conteo, suma y resta con la implementación de los materiales didácticos diseñados con técnicas de gamificación. Encuestas a los profesores para saber si ellos pudieron observar cambios en los niños con respecto a los conocimientos con que se trabajó (conteo, suma y resta). Y finalmente el análisis de diferencia de medias y prueba psicométrica t students de dos muestras emparejadas, de la variable Habilidades para el Cálculo con la variable independiente implementación de materiales educativos gamificados.

\section{RESULTADOS}

A continuación, se describe la implementación de los diferentes recursos que se trabajaron con tres estudiantes con Síndrome de Down: Diego, Mayra y Marilyn. Cabe indicar que se trabajó de manera individual con cada uno de los alumnos. Se utilizaron: tableta, teléfono inteligente (iPhone) y computador (PC) de la investigadora, ya que, aunque la escuela contaba con computadores, estas no estaban actualizadas, y no eran totalmente funcionales.

Los resultados específicos, luego de aplicada la Escala de inteligencia a las personas que participan en la investigación se exponen en la siguiente tabla.

Tabla 3. Resultados de la Escala de Inteligencia de L.M. Terman y M.A. Merrill

\begin{tabular}{|c|c|c|c|c|}
\hline Nombres & Grado/Año & $\begin{array}{c}\text { Edad } \\
\text { Cronológica } \\
\text { (años) }\end{array}$ & $\begin{array}{l}\text { Edad } \\
\text { Mental } \\
\text { (años) }\end{array}$ & Observaciones \\
\hline Diego & $9 .^{\circ}$ & 22 & 6 & $\begin{array}{l}\text { Problemas en discriminar materiales, } \\
\text { diferencias, analogías opuestas, } \\
\text { narraciones, repetición de oraciones y } \\
\text { comprensión de preguntas. }\end{array}$ \\
\hline Mayra & $7 .^{\circ}$ & 14 & 4.5 & $\begin{array}{l}\text { Problemas en comprensión, analogías } \\
\text { opuestas, narraciones. }\end{array}$ \\
\hline Marilyn & $5 .^{\circ}$ & 9 & 3 & $\begin{array}{l}\text { Problemas en repetición de frases, } \\
\text { repetición de dígitos y comprensión. }\end{array}$ \\
\hline
\end{tabular}

En el siguiente diagrama se puede observar claramente cuáles son las habilidades logradas por los alumnos y cuales requieren atención emergente. Por consiguiente, facilita la programación y selección de experiencias de aprendizaje como la graduación que se debe 
realizar según el perfil de los alumnos (Riquelme, 2003). Los resultados específicos, luego de aplicado el Test de Habilidades Básicas para el Cálculo (Pretest) se exponen en el siguiente gráfico.

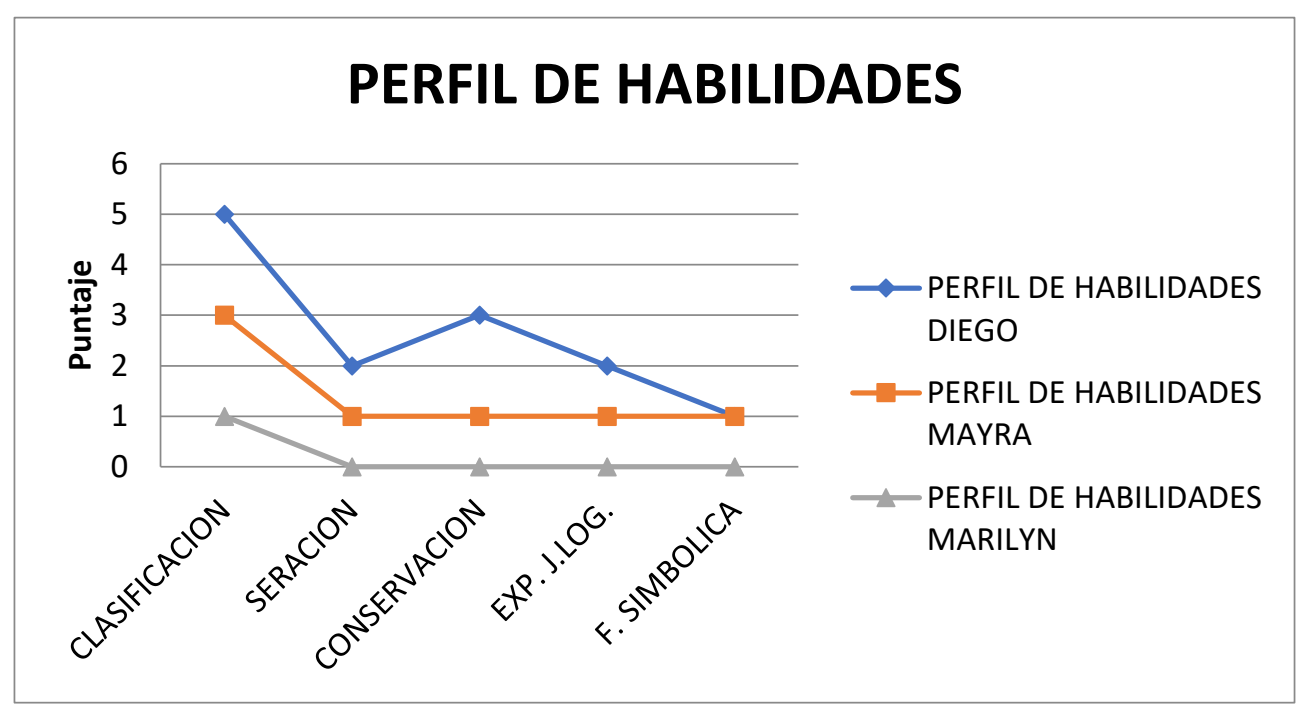

Figura 1. Perfil de habilidades.

\subsection{Caso de estudio: Diego}

\subsubsection{Resultados de las pruebas Pretest y Postest de Habilidades para el Cálculo (HC)}

Al comparar las medias estadísticas de ambas pruebas (pretest y postest) de HC, se observa que aumentaron los valores obtenidos a la prueba de pretest, en todas las habilidades, aunque se ubica al nivel medio alto para la habilidad de clasificación, seriación, expresión de juicio lógico y función simbólica, a excepción de la función de conservación que se ubicó en el nivel medio bajo (véase Figura 2).

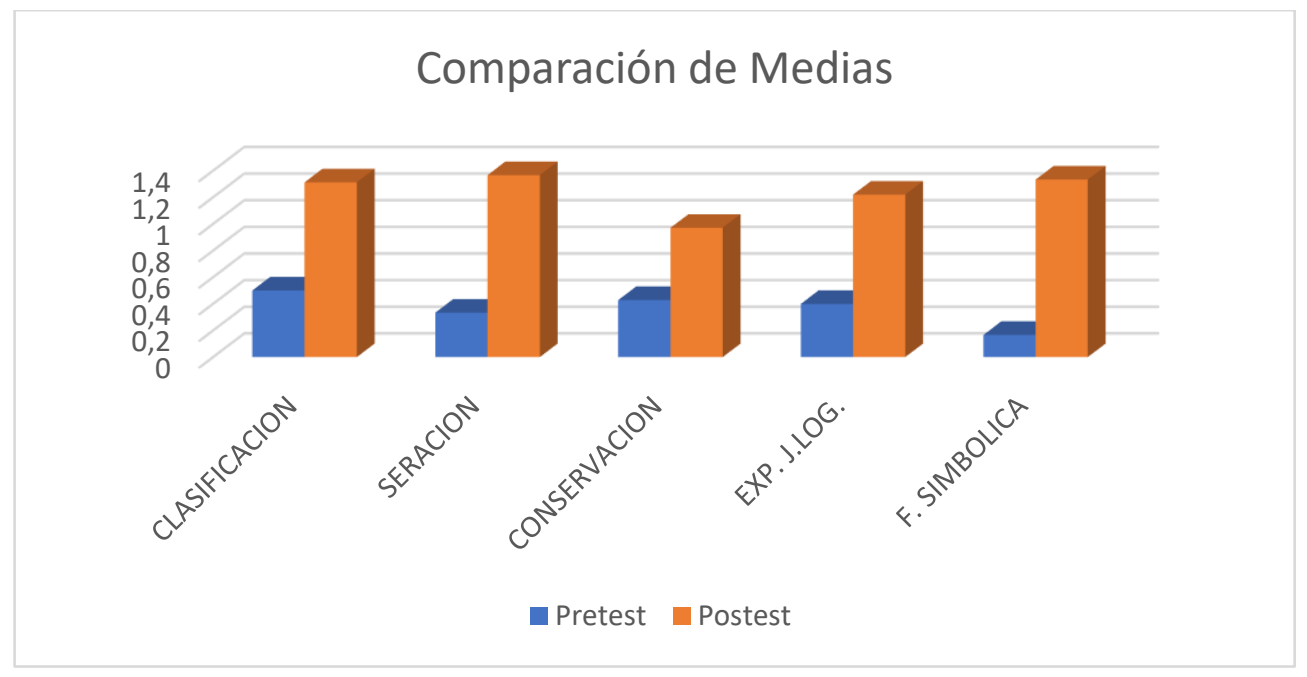

Figura 2. Comparación de medias estadísticas de los resultados pre y postest

Para analizar si las diferencias obtenidas entre las dos pruebas (pretest y postest) de HC fueron significativas, se realizó la prueba $t$ de student. Los resultados muestran diferencias estadísticamente significativas entre las pruebas pretest y postest de la habilidad de clasificación $(p=0.000053)$, seriación ( $p=0.0025)$, juicio lógico y función simbólica; sin embargo, no muestran diferencias estadísticamente en la habilidad de conservación $(p=0.050)$.

\subsection{Caso de estudio: Mayra}




\subsubsection{Resultados de las pruebas Pretest y Postest de Habilidades para el Cálculo (HC)}

Al comparar las medias estadísticas de ambas pruebas (pretest y postest) de HC, se observa que aumentaron los valores obtenidos a la prueba de pretest, en todas las habilidades, aunque se ubica al nivel medio alto para la habilidad de conservación y expresión de juicio lógico, a excepción de la función de clasificación, seriación y función simbólica que se ubicó en el nivel medio o medio bajo (véase Figura 3).

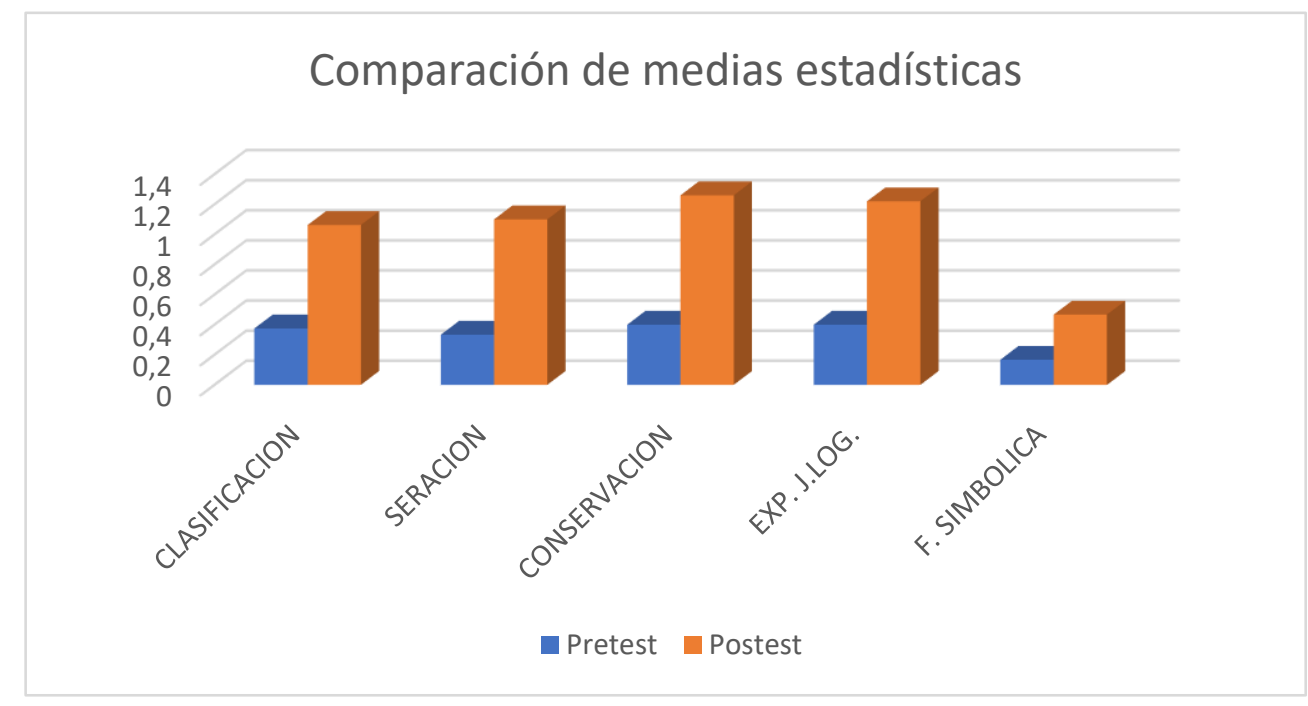

Figura 3. Comparación de medias estadísticas de los resultados pre y postest

Para analizar si las diferencias obtenidas entre las dos pruebas (pretest y postest) de HC fueron significativas, se realizó la prueba t de student. Los resultados muestran diferencias estadísticamente significativas entre las pruebas pretest y postest de las habilidades de clasificación $(p=0.0043)$, no así en las habilidades de seriación $(p=0.032)$, juicio lógico $(p=0.045)$, función simbólica $(p=0.17)$ y en la habilidad de conservación $(p=0.084)$.

\subsection{Caso de estudio: Marilyn}

\subsubsection{Resultados de las pruebas Pretest y Postest de Habilidades para el Cálculo (HC)}

Al comparar las medias estadísticas de ambas pruebas (pretest y postest) de HC, se observa que aumentaron los valores obtenidos a la prueba de pretest, en todas las habilidades, aunque se ubica al nivel medio para la habilidad de clasificación, a excepción de la función de conservación, seriación, expresión de juicio lógico y función simbólica, que se ubico en el nivel medio bajo, (vease Figura 4).

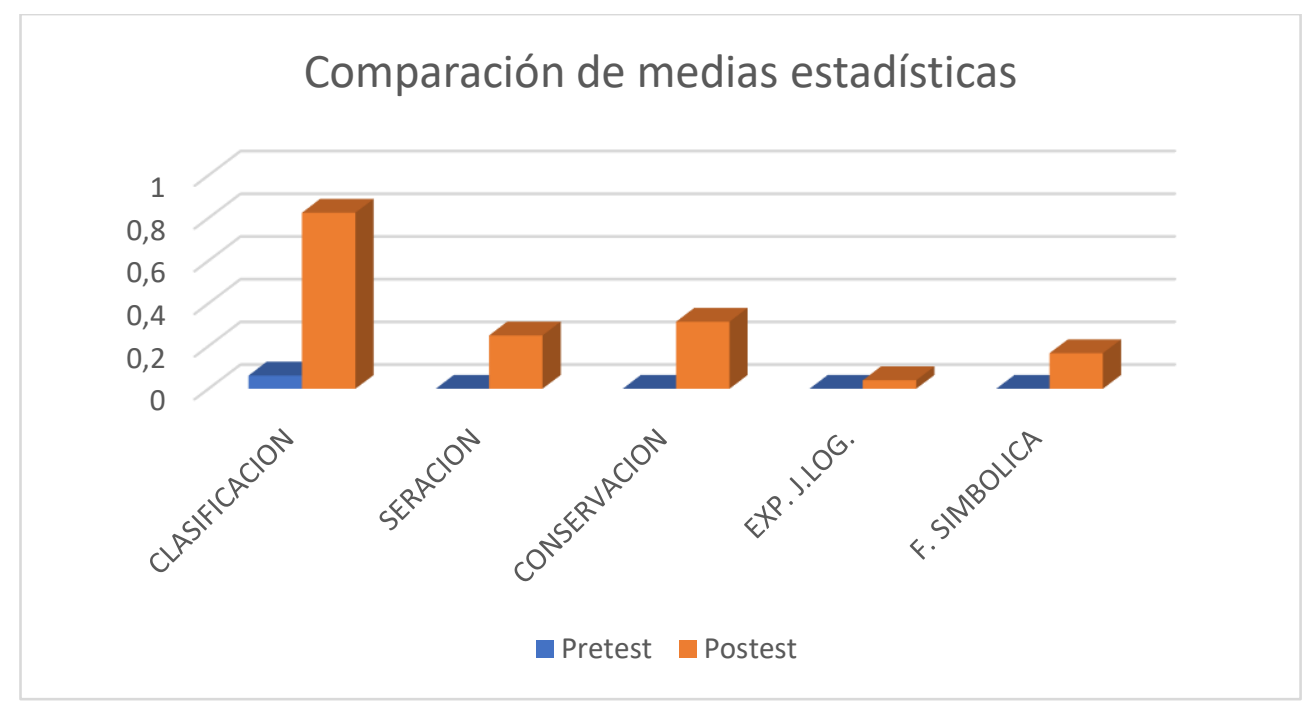


Figura 4. Comparación de medias estadísticas de los resultados pre y postest

Para analizar si las diferencias obtenidas entre las dos pruebas (pretest y postest) de HC fueron significativas, se realizó la prueba t de student. Los resultados muestran diferencias estadísticamente significativas entre las pruebas pretest y postest de las habilidades de clasificación ( $p=0.015)$, no así en las habilidades de seriación $(p=0.036)$, juicio lógico $(p=0.045)$, función simbólica $(p=0.17)$ y en la habilidad de conservación $(p=0.036)$.

\section{DISCUSIÓN Y CONCLUSIONES}

\subsection{Conclusiones}

El análisis comparativo del posible cambio significativo en las Habilidades para el Cálculo, tras la aplicación de los materiales educativos gamificados, aumentaron en puntuación en todas las habilidades, evidenciándose un aumento significativamente superior en las habilidades de clasificación, seriación, siendo lo suficientemente significativo para mostrar una diferencia destacada en general, por lo que la hipótesis formulada en este aspecto se acepta (Hipótesis 0).

Los resultados encontrados en la evaluación final confirman, que en términos generales, los alumnos con Síndrome de Down demostraron mejor del rendimiento en el aprendizaje de conocimientos y habilidades relacionadas con las habilidades de clasificación, seriación, conservación, expresión de juicio lógico y función simbólica, aunque los mejores resultados están en las habilidades de clasificación y seriación, no así, en la expresión de juicio lógico y función simbólica donde se observa una mejora moderada.

Por lo tanto, se ha confirmado que el uso de la gamificación en las aulas, como elemento favorecedor y potenciador del aprendizaje del alumnado, permite el logro de la equidad educativa; además es una herramienta a disposición de los docentes, que aunque falten avances para la correcta aplicación en el aula (formación del profesorado, dotación de recursos y herramientas), animan a los docentes a emplear la gamificación en cualquier etapa para un favorecimiento del aprendizaje sin exclusión (Jiménez, et al., 2019).

Finalmente, el uso del test de habilidades básicas para iniciación al cálculo nos ha ayudado a evaluar los conocimientos de las personas con Síndrome de Down sobre el sistema de numeración decimal, permitiendo observar sus logros y sus deficiencias. Ello, junto con las características cognitivas específicas, nos permitirá diseñar secuencias de aprendizaje individualizadas, que les ayuden a los alumnos investigados a superar sus deficiencias y trabajar los aspectos necesarios para lograr un avance en la comprensión del sistema decimal y de las destrezas adaptadas a sus necesidades educativas especiales.

\subsection{Propuesta de Mejora}

En relación a las conclusiones basadas en los resultados más bajos relacionados con el impulso y fortalecimiento del uso de materiales educativos gamificados; y, una vez que los docentes son conscientes de que la rentabilidad de las TIC no solo depende del propio dispositivo, sino de su adecuada utilización, tanto del profesorado, del alumnado y de las familias, se recomienda implementar las buenas prácticas para la inclusión educativa y el aprendizaje de personas con Síndrome de Down sugeridas en Down España (2012), mismas que se detallan a continuación:

Utilizar los mismos contenidos curriculares que el resto de los alumnos, incorporando las adaptaciones necesarias para su aprendizaje; implicar a las familias en el aprendizaje de sus hijos y en la práctica de las nuevas tecnologías; trabajar en la asociación, la familia y el centro educativo de forma cohesionada con el fin de optimizar el aprendizaje de la persona con síndrome de Down; favorecer el aprendizaje cooperativo en el aula, a través de grupos reducidos de trabajo que compartan objetivos y fomenten la socialización a través del juego; Apoyar gradualmente a la persona con síndrome de Down a través de una ayuda inicial y una vez adquiridos los conocimientos ir retirando 
los apoyos poco a poco, fomentando así la responsabilidad y el trabajo autónomo del alumno; utilizar las tableta para desarrollar las habilidades y capacidades cognitivas necesarias para desarrollar con éxito el proceso educativo: memoria, agilidad mental, atención, concentración, razonamiento, motricidad fina y coordinación visomotora; utilizar las tabletas para exposiciones públicas del alumnado, mejorando la comunicación oral y la organización de su pensamiento; abrir el aula al uso de recursos existentes en otros medios, bibliotecas virtuales, blogs, páginas webs, foros, redes sociales, etc.; personalizar las aplicaciones que lo permitan, incluyendo imágenes de su vida diaria como aprendizaje significativo y como generalización de sus aprendizajes. (p. 17)

\subsection{Logros y Limitaciones}

En general los resultados son positivos pues mejoran el ambiente de aprendizaje; facilitan el intercambio de experiencias e ideas; y favorecen el desarrollo creativo y cognitivo. Sin embargo, se destacan dos variables que son importantes, por un lado, el apoyo familiar y su nivel socioeconómico, pues es una ventaja que los estudiantes ya usen herramientas tecnológicas y por otro lado la atención temprana (Vidal, et al., 2018)

La elección de las aplicaciones seleccionadas se debe a que son gratuitas, interactivas, multimedia y divertidas, ya que cuentan con una variedad de juegos y se centran en trabajar principalmente la capacidad de síntesis, la abstracción, asociación, la función simbólica y la resolución de problemas. Ya que como lo señalan Molina y Santiago (2016), a través del juego los estudiantes no aprenden solo conceptos, sino que es una fuente para el fomento de la cooperación, un método para crear compañerismo en el ámbito escolar, mediante el aprenden a respetar reglas y crean situaciones idóneas para trabajar la inteligencia emocional y social.

La gamificación como metodología se ha revelado como herramienta muy útil como recurso didáctico por dos razones: porque independientemente de lo que enseñan, aúnan el aprendizaje con la diversión y el disfrute, y porque incorporan principios de aprendizaje (Barros, 2016).

La investigación que aquí se ha presentado, a pesar de haber sido un estudio con una población muy pequeña, es el inicio para futuras investigaciones en el Ecuador. En primer lugar, es importante probar los materiales educativos seleccionados con otras poblaciones y con mayor número de alumnos con o sin necesidades educativas especiales; a fin de determinar si es consistente, para continuar con programas de intervención que ayuden a ampliar el conocimiento de las personas con Síndrome de Down y superar las dificultades de estas personas en el aprendizaje matemático.

La elaboración de esta investigación ha permitido integrar conocimientos y enfrentar la complejidad de formular juicios a partir de una información que, siendo incompleta o limitada, incluye reflexiones sobre las responsabilidades sociales y éticas vinculadas a la aplicación de mis conocimientos y juicios de valor.

En cuanto a las limitaciones, somos conscientes que existe una gran variedad de aplicaciones de matemática para tableta, PC e iPhone. Muchas de ellas cumplen objetivos muy diversos para el aprendizaje de las habilidades de clasificación, seriación, conservación, expresión de juicio lógico y función simbólica, en el aprendizaje de la matemática que no están al alcance de las instituciones educativas, por lo que es importante considerar aquellas que se han utilizadas en esta prueba piloto y aquellas que han sido valoradas positivamente por los profesionales educativos de las asociaciones y de los centros educativos del Ministerio de educación del Ecuador.

En lo referente al número de participantes, se considera que la muestra fue bastante pequeña, por lo que es necesario que en investigaciones posteriores se aumente o a su vez se realice la intervención en dos grupos homogéneos (grupo experimental y grupo de control) que permitirá realizar una evaluación del impacto de la implementación de los recursos y la comparación entre los resultados de ambos sea equilibrada.

El tiempo de intervención y la falta de seguimiento, son aspectos a considerar ya que al tratarse de estudios realizados en contexto natural (Escuela de Educación Básica Especializada) no se puede concluir que la educación del Ecuador se sustente en el principio de inclusión, 
aspecto que también se considera una limitación para la investigación: "debería de haberse realizado un seguimiento mucho más prolongado del recurso” (Pareja, 2015, p.31).

\section{ENLACES}

Enlace a los instrumentos: https://bit.ly/2WKGvir

\section{REFERENCIAS BIBLIOGRÁFICAS}

Alonso, F. (2016). Software de desarrollo de destrezas específicas para personas con Síndrome de Down [Trabajo Fin de Grado, Universidad Autónoma de Madrid]. Repositorio UAM. https://bit.ly/350vBw5

Angulo, M. C., Gijón, A., Luna, M., \& Prieto, I. (2006). Manual de atención al alumnado con necesidades específicas de apoyo educativo derivadas de síndrome de Down. Junta de Andalucía. https://bit.ly/3clYU5z

Antequera, M., Bachiller, B., Calderón, M. T., Cruz, A., Cruz, P. L., García, F. J., Luna, M., Montero, F., Orellana, F. M., Reyes, G. (2008). Manual de atención del alumnado con necesidades específicas de apoyo educativo derivadas de dicapacidad intelectual. Junta de Andalucia. https://bit.ly/2S3A1cW

Barros, M. (2016). La gamificación en el aula de lengua extranjera. En J. Lloret (Ed.), El español como lengua extranjera en Portugal II: retos de la enseñanza de lenguas cercanas (pp. 5-14). Secretaría General Técnica. https://bit.ly/3dCUFZP

Bonilla, J. (2012). Actividades Computacionales de Conteo Matemático para Niños con Síndrome de Down [Tesis de maestría, Centro de Investigación y de Estudios Avanzados del Instituto Politécnico Nacional]. https://bit.ly/3cnUL7f

De Benito, B. \& Salinas, J.M. (2016). La investigación basada en diseño en Tecnología Educativa. RIITE. Revista Interuniversitaria de Investigación en Tecnología Educativa, 0, 44-59. http://dx.doi.org/10.6018/riite/2016/260631

Down España. (2012). Proyecto H@z Tic - Guía práctica de aprendizaje digital de lectoescritura mediante tablet para alumnos con síndrome de Down. https://bit.ly/3bBRYqA

Federación Andaluza de asociaciones para el Síndrome Down. (2012). Guía para la atención educativa a los alumnos y alumnas con síndrome de Down. Servicio de Información sobre Discapacidad. https://bit.ly/2yCovyf

Llorens Largo, F., Gallego-Durán, F. J., Villagrá-Arnedo, C. J., Compañ, P., Satorre Cuerda, R., \& Molina-Carmona, R. (2016). Gamificación del proceso de aprendizaje: lecciones aprendidas. Revista VAEP-RITA, 4, 25-32. https://bit.ly/2Vy8HWv

Molina, M., \& Santiago, A. (2016). Animar a la lectura de textos multimodales a través del juego: cuaderno didáctico de Hervé Tullet. En A. Díez, V. Brotons, D. Escandell y J. Rovira (Eds.), Aprendizajes plurilingües y literarios, nuevos enfoques didácticos (pp. 538-549). Publicacions de la Universitat d'Alacant. https://bit.ly/2LjFIQ1

Pareja, D. (2015). Gamificación \& Síndrome Down [Trabajo Fin de Grado, Universidad de Málaga]. https://bit.ly/35MUuZb

Riquelme, G. (2003). Test de habilidades básicas para la iniciación al cálculo "TIC". Revista Enfoques Educacionales, 5, 137-156. https://bit.ly/2Y1CHvw

Ruiz, E. (2012). Programación educativa para escolares con síndrome de Down. Fundación Iberoamericana Down21. https://bit.ly/3aCqC2d

Tangarife, D., \& Jaramillo, J. (2015). Diseño de una aplicación para enseñar las operaciones básicas de las matemáticas a personas con Síndrome de Down [Trabajo Fin de Máster,Universitat, Oberta de Catalunya]. https://bit.ly/2zvD4ny

Vidal, M. I., López , M., Marín, D., \& Peirats, J. (2018). Revisión y Análisis de investigación publicada sobre intervención gamificada en discapacidad intelectual. Revista Científica 
de Educación y Comunicación en la Sociedad del Conocimiento, 2(18), 274-297. https://bit.ly/3awSftF

Zicherman, G., \& Cunningham, C. (2011). Gamification by design. O'Reilly Media. https://bit.ly/3csbl0A

\section{INFORMACIÓN SOBRE LOS AUTORES}

\section{Nancy Castillo Jumbo}

Universitat Rovira i Virgili

Master en Tecnología Educativa: E-Learning y Gestión del Conocimiento por Universitat Rovira i Virgili-España (2019); Magister en Desarrollo de la Inteligencia y Educación por UTPL (2010); Ingeniero en Ciencias de la Computación e Informática (2005). Auditora Educativa de Zona 7 Educación. Miembro del Equipo Investigador de Auditores de Zona7 (2019). Rectora Instituto Superior Pedagógico "Ciudad de Cariamanga" (2008). Experiencia en docencia en Educación Superior de 11 años. Líneas de investigación: Diversidad, inclusión y tecnología, Estándares Educativos y Efectos Escolares. Email: nancy.castillo@educacion.gob.ec

\section{José Jiménez González}

Universitat Rovira i Virgili

Doctor Ph.D en Pedagogía por la Universitat Rovira i Virgili-España (2001); Master en Tecnología Educativa: Diseño de Materiales y Entornos de Formación (Pedagogía) por URV-España (2001); Licenciado de Grado en Pedagogía por URV (1995). Docente Investigador del Departamento de Pedagogía. Líneas de investigación: Investigación aplicada en Educación y Teconología. Email: josemiguel.jimenez@urv.cat

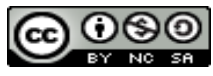

Los textos publicados en esta revista están sujetos a una licencia de Reconocimiento 4.0 España de Creative Commons. Puede copiarlos, distribuirlos, comunicarlos públicamente y hacer obras derivadas siempre que reconozca los créditos de las obras (autoría, nombre de la revista, institución editora) de la manera especificada por los autores o por la revista. La licencia completa se puede consultar en:Licencia Creative Commons Atribución-NoComercial-Compartir por igual 4.0 Internacional.
} 